Draft Version November 3, 2018

Preprint typeset using $\mathrm{I}_{\mathrm{A}}^{\mathrm{T}} \mathrm{EX}$ style emulateapj v. 03/07/07

\title{
*IMPACTS OF A SUPERSONIC SHOCK FRONT ON STAR FORMATION IN THE BULLET CLUSTER
}

\author{
Sun Mi Chung ${ }^{1}$, Anthony H. Gonzalez ${ }^{1}$, Douglas Clowe ${ }^{2}$, Dennis Zaritsky ${ }^{3}$, Maxim Markevitch ${ }^{4}$, Christine \\ JONES $^{4}$ \\ Draft version November 3, 2018
}

\begin{abstract}
We use the Bullet Cluster (1E0657-56) to investigate the extent to which star formation in cluster galaxies is influenced by ram pressure from supersonic gas (Mach 3) during a cluster merger. While the effects of ram pressure have been studied for individual galaxies infalling into galaxy clusters, this system provides a unique opportunity to investigate the impact of dramatic merger events on the cluster galaxy population. In this analysis we use Spitzer IRAC data to study star formation. At the redshift of the cluster the $6.2 \mu \mathrm{m} \mathrm{PAH}$ feature is redshifted into the $8 \mu \mathrm{m}$ band, enabling use of the $\mathrm{m}_{4.5}-\mathrm{m}_{8}$ color as a proxy for specific star formation rate. We find that the color distribution on the two sides of the shock differ by less than $2 \sigma$, and conclude that ram pressure from the shock front has no dramatic, immediate impact on the star formation of cluster galaxies in the Bullet Cluster.
\end{abstract}

Subject headings: galaxies: clusters: individual (1E0657-56, The Bullet Cluster) - galaxies: evolution

\section{INTRODUCTION}

It has long been observed that there is a correlation between galaxy morphology and local density (Dressler 1980), known as the density-morphology relation. Although there are various physical mechanisms that can transform star-forming late-type galaxies into quiescent early-type galaxies, it is unknown whether this transformation occurs mostly in the galaxy group or galaxy cluster environment.

Preprocessing of galaxies in the group environment has been argued by some (Zabludoff \& Mulchaey 1998; Kodama et al. 2001) to be a principal mechanism driving the predominance of the passive galaxy populations observed in galaxy clusters. In galaxy groups, the velocity dispersions are sufficiently low that processes such as galaxy-galaxy interactions can quench star formation and morphologically alter galaxies. In addition to galaxy-galaxy interactions, Kawata \& Mulchaey (2008) show that "strangulation" is an important mechanism that leads to quenched star formation in the group environment. Using a cosmological chemodynamical simulation, Kawata \& Mulchaey (2008) show that most of the hot gas in a disk galaxy is stripped away, thus cutting off a source of new cold gas (some of the hot gas cools to form cold gas), which is necessary to maintain star formation.

Others have argued that cluster-specific processes are responsible for the high early-type fraction. The simulations of Berrier et al. (2008) argue that the transformation of late-type to early-type galaxies is mostly attributed to processes internal to the galaxy cluster environment, rather than the group environment.

* THIS PAPER INCLUDES DATA GATHERED WITH THE 6.5 METER MAGELLAN TELESCOPES LOCATED AT LAS CAMPANAS OBSERVATORY, CHILE.

${ }^{1}$ Department of Astronomy, University of Florida, Gainesville, FL 32611-2055; schung@astro.ufl.edu

2 Department of Physics and Astronomy, Ohio University, 251B Clippinger Lab, Athens, OH 45701

3 Steward Observatory, University of Arizona, 933 North Cherry Avenue, Tucson, AZ 85721

${ }^{4}$ Harvard-Smithsonian Center for Astrophysics, 60 Garden Street, Cambridge, MA 02138
Berrier et al. (2008) find that most of the galaxies in a galaxy cluster are accreted directly from the field, rather than from infalling galaxy groups. In this case, mechanisms such as galaxy harassment (Moore et al. 1996) and ram pressure stripping (Gunn \& Gott 1972) that are most efficient in massive clusters must drive this transformation.

Simulations have shown that ram pressure stripping can remove some or all of the interstellar medium (ISM) from late-type galaxies as they enter the cluster potential (Roediger \& Hensler 2005; Mayer et al. 2006; Brüggen \& De Lucia 2008). Studies of galaxies in clusters with observed HI deficiencies or truncated disks support the idea that ram pressure stripping can transform a gas-rich spiral galaxy into an anemic spiral galaxy (e.g. Vollmer et al. 2001, 2008), which may eventually evolve into a lenticular (e.g. Bekki et al. 2002, but see Boselli \& Gavazzi (2006)). Ram pressure has also been shown to trigger bursts of star formation in cluster galaxies (Gavazzi et al. 2001, 2003), and recent simulations (Kronberger et al. 2008) predict enhanced star formation rates in galaxies undergoing a ram pressure event.

While previous studies have examined the impact of ram pressure on individual galaxies falling into the cluster potential (Cortese et al. 2007; Chung et al. 2007) the Bullet Cluster provides a unique opportunity to examine the effect of ram pressure induced by cluster mergers. Although galaxies in any cluster environment undergo ram pressure as they travel through the ICM, galaxies in a major merger event such as those we observe in the Bullet Cluster will experience a dramatic enhancement in the pressure since $P \propto V^{2}$. It is thus possible that this brief transient phase may have a significant impact on the properties of the cluster galaxy population. The Bullet Cluster is an ideal site in which to quantify the importance of such merger-induced ram pressure.

In this paper we conduct an initial exploration of the impact of the shock front upon polycyclic aromatic hydrocarbon (PAH) emission, and hence star formation, in cluster galaxies. We analyze Spitzer data taken with the Infrared Array Camera (IRAC; Fazio et al. 2004), in conjunction with optical spectra observed with 
the Inamori Magellan Areal Camera and Spectrograph (IMACS; Bigelow et al. 1998).

\section{GEOMETRY AND CLUSTER PROPERTIES}

The Bullet Cluster, at a redshift of $z=0.296$, is a head-on collision between two clusters of unequal mass, which we call the main cluster and subcluster. Markevitch et al. (2002) [M02] observed a sharply defined bow shock front, driven by the subcluster and propagating in the gas of the main cluster with a velocity of $4740_{-550}^{+710} \mathrm{~km} \mathrm{~s}^{-1}$ (Mach number $M=3.0_{-0.35}^{+0.45}$ ) (Markevitch et al. 2008, in preparation). Hydrodynamic simulations showed that the subcluster itself has a lower velocity relative to the main cluster, $\sim 2700 \mathrm{~km} \mathrm{~s}^{-1}$ (Springel \& Farrar 2007; see also Milosavljević et al. 2007). While the subcluster and the shock front might be expected to move together, the gravity of the subcluster causes the main cluster's gas in front of the shock to flow onto the subcluster. As a result, the shock front has a higher velocity in the reference frame of that gas inflow, which is what is measured in X-rays. Markevitch et al. (2004) constrain the inclination angle of the collision, with respect to the plane of the sky, to $i<8^{\circ}$, based on the Mach number of the shock and subcluster relative line-of-sight velocity $\left(\sim 600 \mathrm{~km} \mathrm{~s}^{-1}\right)$ (Barrena et al. 2002). From the velocity and geometry of the system, it is computed that the subcluster exited the main cluster core $\sim 0.15 \mathrm{Gyr}$ ago.

The simple geometry of the Bullet Cluster makes it an ideal system in which to study how galaxy properties such as star formation are affected by ram pressure. Because the merger is nearly in the plane of the sky, the velocity of galaxies with respect to the intracluster medium (ICM) is well constrained, providing a good quantitative measure of the ram pressure exerted by the gas on the galaxies. In addition, the recentness of the collision ( 0.15 Gyr) allows us to use sensitive indicators of recent star formation, direct and indirect measures of the ionizing flux from $\mathrm{O} / \mathrm{B}$ stars with lifetimes of 0.1 Gyr.

\section{PHYSICAL ASSUMPTIONS}

The analysis in this paper requires several assumptions related to the star formation rate and the three-space galaxy distributions for the main cluster and subcluster.

\subsection{Star Formation Rate}

To probe star formation rates, we use the PAH emission at rest wavelength $\lambda=6.2 \mu \mathrm{m}$, which at the cluster redshift is observed in the $8 \mu \mathrm{m}$ IRAC band. Ultraviolet (UV) photons that are produced by young hot stars are known to excite PAH molecules which then emit in the $\lambda=6.2 \mu \mathrm{m}$ and other infrared bands (Leger \& Puget 1984; Allamandola et al. 1985). More recent studies have shown that the $6.2 \mu \mathrm{m}$ luminosity is correlated with total infrared luminosity (Peeters et al. 2004; Brandl et al. 2006), which is a tracer of star formation rate (Kennicutt 1998). However, PAH emission is not a unique signature of star formation, which can also be excited by visible photons (Uchida et al. 1998; Li \& Draine 2002), as well as by active galactic nuclei (AGN) (Freudling et al. 2003). It has also been shown that PAH emission is dependent on metallicity (Engelbracht et al. 2005; Smith et al. 2007) and thus not a robust star formation rate indicator for galaxy samples that span a large range in metallicity (Boselli et al. 2004).

For the Bullet Cluster, both effects are expected to be second order. Our data are negligibly affected by AGN contamination (see $§ 5$ ), and our sample is dominated by massive galaxies, for which the metallicity variations are not dramatic. Moreover, the metallicity distribution should be the same for galaxies on both sides of the shock. The PAH flux should nevertheless be considered a rough proxy for star formation rate rather than a precision tracer.

To normalize the $8 \mu \mathrm{m}$ flux, we use the $4.5 \mu \mathrm{m}$ flux as a proxy for mass. The $4.5 \mu \mathrm{m}$ flux originates mostly from the old red giant stars, and probes the stellar mass of a galaxy at the cluster redshift. By using the ratio of $8 \mu \mathrm{m}$ to $4.5 \mu \mathrm{m}$ flux or $\mathrm{m}_{4.5}-\mathrm{m}_{8}$ color, we can roughly trace star formation rate per stellar mass. Specifically, $8 \mu \mathrm{m}$ emission in excess of that expected from the RayleighJeans tail of the cold stellar component is assumed to arise from star-formation induced PAH emission. We estimate the level of impact that the $\mathrm{M} \sim 3$ shock has had on star formation activity in individual galaxies by looking at the $\mathrm{m}_{4.5}-\mathrm{m}_{8}$ color as a function of distance from the shock front.

\subsection{Galaxy Distribution and Projection}

We proceed with our analysis under the simplifying assumption that galaxies that lie ahead of the shock front are main cluster "pre-shock" galaxies, and those behind the shock front are main cluster "post-shock" galaxies (Figure 11). The post-shock galaxies have already experienced the ram pressure exerted by the gas behind the shock, whereas the pre-shock galaxies have not yet been affected. The true physical situation is clearly more complex, with contamination from the subcluster galaxies as well as background/foreground sources not associated with the Bullet Cluster. Although there is some contamination from the subcluster galaxies, we assume that the galaxy populations on both sides of the shock front are dominated by main cluster galaxies based on estimates of the total stellar mass. The stellar mass of the main cluster is approximately an order of magnitude larger than the stellar mass of the subcluster.

We also note that any effect of contamination from the subcluster galaxies will be strongest at the shock front, which bisects the subcluster brightest group galaxy. Subcluster galaxies just ahead of the shock front experience a strong ram pressure as they collide with the main cluster ICM at $\sim 4700 \mathrm{~km} \mathrm{~s}^{-1}$, whereas subcluster galaxies just behind the shock are somewhat shielded by the shock front and feel a much weaker ram pressure with a slower gas inflow velocity of $\sim 1600 \mathrm{~km} \mathrm{~s}^{-1}$. Therefore any effect of ram pressure on star formation rate has the opposite sign for subcluster galaxies near the shock front, compared to the main cluster galaxies.

We assume that there is a sharp division between postshock and pre-shock galaxies, where all galaxies "behind" the shock front (leftward of the curve in Figure 1) have experienced the effects of the shock. However, because of the projection of galaxies and curvature of the shock front surface along the line of sight, there is a gradual transition from post-shock to pre-shock galaxies. This projection effect softens any step-like increase/decrease in color (or star formation rate) across the shock boundary. By 
generating galaxies in a random distribution according to the Navarro, Frenk, and White (NFW) (Navarro et al. 1997) profile, and assuming the shock front is an axisymmetric cone projected in the plane of the sky and spanning $\sim 550 \mathrm{kpc}$ from the axis of symmetry, we estimate that the transition region during which the observed galaxies go from being fully post-shock to fully pre-shock galaxies is $\sim 0.5 \mathrm{Mpc}$.

\section{OBSERVATIONS}

\subsection{IRAC}

Observations of 1E0657-56 were taken on December 17 2004, with Spitzer/IRAC. Data were collected from all four IRAC channels $-3.6 \mu \mathrm{m}, 4.5 \mu \mathrm{m}, 5.8 \mu \mathrm{m}$, and $8 \mu \mathrm{m}$, in full array readout mode. A medium scale, cycling dither pattern was used, with 20 pointings. At 2 frames per pointing and $100 \mathrm{~s}$ exposure time per frame, the total integration time per IRAC band was $4000 \mathrm{~s}$. The native IRAC pixel scale is 1.22 arcsec, but the final reduced images were set to have a pixel scale of 0.86 arcsec pixel $^{-1}$, with an effective field of view (FOV) of $3.7 \times 3.7$ arcmin covered by the four channels. The frames were processed using the Spitzer Science Center (SSC) IRAC Pipeline, and mosaics created from the basic calibrated data (BCD) frames using MOPEX.

\subsection{IMACS}

We analyzed optical spectroscopic data taken with Magellan/IMACS in 2005 and 2006. In 2005, 430 objects were observed with four masks, with exposure times ranging from 4.8 to $5.4 \mathrm{ks}$. During this run, red sequence galaxies were given highest priority as targets. The following year, two additional masks were obtained, with exposure times of 10.8 and $12.0 \mathrm{ks}$. Target priority was given to objects with relatively red $\mathrm{m}_{4.5}-\mathrm{m}_{8}$ colors. The wavelength range of all spectra are $4000 \AA-9000 \AA$, with a dispersion of $2 \AA$ per pixel. We note that spectroscopic selection criteria for galaxies on both sides of the shock front are identical.

\section{DATA ANALYSIS}

\subsection{IRAC Photometry}

Source detection and photometry of the IRAC images are performed with Source Extractor (Bertin \& Arnouts 1996). Sources are identified in the $4.5 \mu \mathrm{m}$ image with a $3.5 \sigma$ detection threshold. Magnitudes are measured within a $5^{\prime \prime}$ aperture. We choose to apply no aperture corrections to the photometry. In this analysis we are primarily interested in colors rather than total magnitudes, which for $\mathrm{m}_{8}-\mathrm{m}_{5.8}$ and $\mathrm{m}_{4.5}-\mathrm{m}_{3.6}$ would change by only $\sim 0.05$ magnitudes with the corrections applied. Since the corrections themselves are uncertain by up to $10 \%$ (http://ssc.spitzer.caltech.edu/irac/calib/extcal), excluding systematics, we use raw aperture magnitudes for all analyses. In addition, aperture corrections are expected to be minor when examining colors rather than absolute magnitudes.

\subsection{IRAC Sample Selection}

The curvature of the shock contour indicates that the direction of propagation lies within $\sim 5$ degrees of the east-west axis (M02). The shock front was defined as the X-ray brightness contour whose most westward point lies at 104.6 degrees in right ascension (Figure 1), with a maximum and minimum declination of -55.908 and 55.985 degrees. Objects located above or below (north or south) the shock front were not included in our analyses, since these sources are not in the direct path of the shock front and thus should be minimally affected by the propagating shock. We limit our analyses to the region of spatial overlap between the four IRAC bands, detecting a total of 758 sources within this "analysis region".

We eliminate unsaturated stars from the IRAC catalog using Magellan optical photometry (Clowe et al. 2006). In the inner region of the R-band image where the point spread function (PSF) is small $(\sim 0.45$ arcsec FWHM), objects with a half-light radius of 2.6 to 3.2 pixels $(0.111$ arcsec/pixel) and an R-band aperture magnitude of 18 to 24.1 (with a 7.8 pixel aperture), are flagged as stars. Due to a gradient of the PSF in the image, half-light radius and aperture magnitude cuts are scaled accordingly to provide a catalog of unsaturated stars. Saturated stars are identified manually by inspecting the Magellan Rband image to look for saturated objects.

To minimize contamination from bright foreground galaxies, we impose a magnitude limit of $\mathrm{m}_{4.5}>14.20$ - the magnitude of the brightest cluster galaxy (BCG) in the main cluster. We also apply a magnitude cut of $\mathrm{m}_{4.5}<17$, which is sufficiently faint to detect most of the $8 \mu \mathrm{m}$ sources. While going deeper to $\mathrm{m}_{4.5}<18$ increases the number of cluster candidates, we opted for a more conservative $\mathrm{m}_{4.5}<17$ magnitude limit so as to minimize the background contamination. Most importantly, the analyses described in this paper produce the same result within error bars, whether we apply a magnitude limit of $\mathrm{m}_{4.5}<17$ or $\mathrm{m}_{4.5}<18$.

Of the 758 sources, 18 are brighter than the main cluster BCG, 176 are stars, leaving us with 564 sources. Eliminating sources fainter than $\mathrm{m}_{4.5}=17.0$, we are left with 169 sources in the direct path of the shock front and 31 that are above/below the shock front. The distance from likely star-forming galaxies to the shock front is illustrated by arrows in Figure 1 .

\subsection{IMACS Spectroscopy}

To reduce contamination from background and foreground sources, we reduce a total of 458 IMACS spectra with IRAF and IDL, then cross-correlate them with template spectra, using the IRAF task xcsao to obtain redshifts. We use the same templates as Tran et al. (2005), each spectrum representing a different type of galaxy - giant elliptical, E+A, Sb spiral, and an emission line galaxy (ELG). Of the 458 spectra, we obtain reliable redshifts for 326 sources. We augment our spectroscopic data set with 133 redshifts (Barrena et al. 2002, priv. comm), which more densely sample the cluster core, yielding a total of 459 sources with known redshifts.

We next associate the spectroscopic redshifts with IRAC sources for the 114 spectra that lie within the IRAC field-of-view. Of these 114 sources, 67 are cluster members with redshifts that lie within $\pm 2000 \mathrm{~km} \mathrm{~s}^{-1}$ of the mean cluster redshift $(z=0.296)$, and 47 are interlopers. Sixty-three out of the 67 cluster members, and 42 out of the 47 interlopers, meet the $\mathrm{m}_{4.5}<17$ criteria. This leaves us with 134 "cluster candidates", which include all IRAC sources that meet our magnitude criteria, 
are located within the direct path of the shock front, and exclude all known interlopers.

\section{RESULTS AND DISCUSSION}

\subsection{Impact of the Shock}

We use the $\mathrm{m}_{4.5}-\mathrm{m}_{8}$ color to probe relative star formation rates per unit stellar mass. At the redshift of the cluster $(z=0.296)$, the prominent PAH emission band at rest wavelength $6.2 \mu \mathrm{m}$ falls into the $8.0 \mu \mathrm{m}$ IRAC band. PAH emission, which is excited primarily by UV photons, most often originates from photodissociation regions of star formation sites (e.g. Howell et al. 2007). Although $\mathrm{PAH}$ emission is sometimes also associated with AGN (Freudling et al. 2003), only 2 out of 134 cluster candidates in the direct path of the shock front have IRAC colors within the AGN wedge (Lacy et al. 2004; Stern et al. 2005). Both AGN candidates have $\mathrm{m}_{4.5}-\mathrm{m}_{8}<0.6$, and thus do not contribute spurious star formation signatures.

Figure 2 shows color as a function of distance from the shock front for 134 cluster candidates. The dotted horizontal lines show the expected color of an elliptical and Sbc type galaxy at $\mathrm{z}=0.3$ (Assef et al. 2008). These predicted colors come from a set of low-resolution spectral templates, derived by Assef et al. (2008), using optical and near-infrared photometry of over 16,000 galaxies in the NOAO Deep Wide-Field Survey Boötes region, and redshifts from the AGN and Galaxy Evolution Survey (AGES). For elliptical galaxies the $8 \mu \mathrm{m}$ emission comes from the Rayleigh-Jeans tail of the stellar component, for which the $\mathrm{m}_{4.5}-\mathrm{m}_{8} \sim-0.1$ at $\mathrm{z}=0.3$ (Assef et al. 2008). Colors redder than the elliptical locus are indicative of $\mathrm{PAH}$ emission in the $8 \mu \mathrm{m}$ band.

A majority of the IRAC sources have colors consistent with red sequence galaxies in the cluster - only $17 \%$ of the cluster candidates have a color $\mathrm{m}_{4.5}-\mathrm{m}_{8}>0.5$, and $6 \%$ of the sources have $\mathrm{m}_{4.5}-\mathrm{m}_{8}>1$. Although there is a selection effect in favor of red sequence galaxies with the spectroscopic data, Figure 2 uses the entire IRAC sample, for which there is no bias towards quiescent ellipticals.

The spectroscopic data is useful for eliminating interlopers, but the sample of confirmed cluster members (solid points in Figure 2 \& Figure 3) is of limited value in the current analysis because the spectroscopic program preferentially targeted red sequence galaxies. Indeed, only 2 of the 23 cluster candidates with $\mathrm{m}_{4.5}-\mathrm{m}_{8}>0.5$, presently have spectra confirming their membership.

Instead, we focus in the current analysis on the full sample of 134 candidate members and assess whether there is evidence for a correlation between the luminosity-weighted color of cluster members and their distance from the shock front. For each galaxy we compute the distance from the shock (Figure 1), and then compute the integrated color of all galaxies in $275 \mathrm{kpc}$ wide bins (Figure 3). For each bin we use the bootstrap technique with 10,000 realizations to calculate the uncertainties. The dotted lines correspond to the total integrated color of all objects on each side of the shock front, with the width of the shaded grey region corresponding to the $1 \sigma$ confidence interval calculated via the bootstrap method.

For the individual data points on Figure 3, the size of the bootstrap error bars indicates that the mean color is strongly sensitive to the small subset of strongly starforming galaxies. For example, in the bin directly ahead of the shock front, there are 2 (out of 22) galaxies that are responsible for the relatively high integrated color. One of these two galaxies is a spectroscopically confirmed, face-on spiral; the other is a large disk galaxy whose optical color is bluer than the red sequence.

The horizontal dotted-dashed line in Figure 3 shows the predicted color of an elliptical galaxy at $z=0.3$ (Assef et al. 2008). The data shown in Figure 3 are meant to show an excess of color in the post-shock and pre-shock galaxies, in comparison to what one would expect from a purely quiescent elliptical galaxy. Overall, Figure 3 shows that there is no drastic change in color across the shock boundary. The integrated color of the post-shock versus pre-shock galaxies is consistent to within $2 \sigma$.

The above technique is a rather blunt means of assessing the impact of the shock front, given that it includes the red sequence galaxies, where we expect minimal change, as well as the star-forming galaxies. An alternate approach is to use the Kolmogorov-Smirnoff (KS) test to understand how statistically different the populations are on either side of the shock. We first compare the full distributions on the two sides of the shock front. Using all 134 candidate members, a KS-test indicates that the colors on the two sides of the shock differ at only the $1.3 \sigma$ level; consequently, the data do not exclude the hypothesis that the galaxies are all drawn from the same population. We note that the full sample includes elliptical galaxies, whose $\mathrm{m}_{4.5}-\mathrm{m}_{8}$ color simply reflects the cold stellar component of the Rayleigh-Jeans tail rather than star formation. All galaxies are included in our initial KS-test because we are looking for changes in the global color distribution of galaxy colors across the shock front.

To avoid the gas-poor elliptical galaxies whose $\mathrm{m}_{4.5^{-}}$ $\mathrm{m}_{8}$ color is not a good proxy for specific star formation rate, we can exclusively use galaxies whose $\mathrm{m}_{4.5}-\mathrm{m}_{8}$ color is redder than some value. Comparing the color distribution across the shock front for galaxies with $\mathrm{m}_{4.5^{-}}$ $\mathrm{m}_{8}>0.2, \mathrm{~m}_{4.5}-\mathrm{m}_{8}>0.5$, and $\mathrm{m}_{4.5}-\mathrm{m}_{8}>1$, the KS-test indicates that the colors on the two sides of the shock front differ at the $0.8 \sigma, 1.5 \sigma$, and $1.2 \sigma$ level, respectively. Although we avoid the elliptical galaxies by imposing color cuts, we also introduce an uncertainty since objects may move in and out of the sample due to the impact of the shock front, without leaving a known trace on the final color distribution. However, we emphasize that the KS-test for all of our color cuts shows no evidence for significant change in the color distribution from pre-shock to post-shock galaxies.

For comparison, we also investigate the optical properties of our IRAC sample. Figure 4 presents the colormagnitude relation (CMR) for the 88 objects out of the 134 IRAC cluster candidates with $R<20.5$, using our Magellan data and again excluding spectroscopically confirmed interlopers. As indicated by the IRAC colors, Figure 4 confirms that most of the IRAC sources are red sequence galaxies. However, to determine exactly which objects can be classified as part of the red sequence, we need three parameters of the CMR - the slope, zeropoint, and dispersion.

We adopt a CMR slope of -0.076 , derived from $57 \mathrm{X}$ - 
ray clusters examined by López-Cruz et al. (2004), with redshifts ranging from $0.02 \leq \mathrm{z} \leq 0.18$. To obtain the zeropoint of the CMR relation, we fit the data with this fixed slope. This fit yields a zero-point of 3.927 , which is $\sim 0.1$ mag brighter than the expected zero-point calculated from the zero-point-redshift relation of López-Cruz et al. (2004). Finally, we calculate the dispersion by fitting a Gaussian function to the CMR residuals, excluding outliers. We derive $\sigma=0.136 \mathrm{mag}$, indicating that our dispersion is indeed dominated by photometric uncertainty.

We classify all objects within $2 \sigma$ of the CMR as red sequence galaxies, indicated by the dotted lines in Figure 4. Out of the 7 objects with $\mathrm{m}_{4.5}-\mathrm{m}_{8}>0.5$ and $R<20.5,5$ of them lie more than $2 \sigma$ below the CMR (with one object just barely within $2 \sigma$ ). These red IRAC sources consitute $5 / 12$ or $42 \%$ of the objects below the $2 \sigma$ line. The optical data thus confirm that objects with red $\mathrm{m}_{4.5}-\mathrm{m}_{8}$ colors correspond to optically blue and likely star-forming galaxies.

There are a few caveats in interpreting the results discussed above. Foremost, there remains contamination by foreground and background sources for the galaxies that lack spectroscopic confirmation. Foreground interlopers among the star-forming galaxies may depress the observed significance of any color change across the shock front. It is unlikely however that removing these foregrounds will create a significant difference in the mean $\mathrm{m}_{4.5}-\mathrm{m}_{8}$ color across the shock boundary. A total of $55 \%$ of IRAC sources with color $\mathrm{m}_{4.5}-\mathrm{m}_{8}>0.5$ are known interlopers, while the fraction of known interlopers is only $22 \%$ for sources with $\mathrm{m}_{4.5}-\mathrm{m}_{8}<0.5$. Because the fraction of interlopers is higher for late-type galaxies than early-type galaxies, eliminating these objects will likely cause the mean color on both sides of the shock front (Figure 3) to approach the quiescent value.

\subsection{Physical Interpretation}

The IRAC data show no significant change in color across the shock boundary (Figure 3), indicating that the gas behind the shock front does not have a large impact on the current or recent star formation activity in the individual galaxies. One possible explanation is that ram pressure stripping may deplete existing gas reservoirs in the outskirts of galaxies without disturbing current/recent star formation sites that may be more centrally located in the disk. Following Gunn \& Gott (1972), a galaxy moving face-on through an ICM experiences a ram pressure of $P=\rho V^{2}$. If ram pressure exceeds the galaxy's internal gravitational pressure, ram pressure stripping occurs. The gravitational pressure can be expressed as $P=2 \pi G x \frac{M_{s t a r}^{2}}{r_{g}^{4}}$, where $G$ is the gravitational constant, $x$ is the interstellar gas to stellar mass ratio, $M_{s t a r}$ is the stellar mass, and $r_{g}$ is the galaxy radius. A galaxy with stellar mass $M_{\star}=10^{10} M_{\odot}$ and $x=0.6$, should experience ram pressure stripping at a distance of $\sim 4 \mathrm{kpc}$ from its center, assuming that it moves face-on through an ICM of density $4 \times 10^{-3} \mathrm{~cm}^{-3}$, at a velocity of $\sim 2700 \mathrm{~km} \mathrm{~s}^{-1}$. This is somewhat beyond the expected disk scale length for a galaxy with stellar mass $M_{\star}=10^{10} M_{\odot}$ (Pizagno et al. 2005). Therefore, we might not expect to see evidence of disturbed star formation due to ram pressure, since much of the current star formation occurs within the disk scale length. How- ever, the gas density and galaxy velocity (with respect to the ICM) are highly uncertain at any significant distance from the shock front, since both quanities change with time and position in the cluster.

If ram pressure stripping destroys gas reservoirs in galaxies that host significant star formation, we might expect to see an excess of post-starburst galaxies in $\sim 10$ Myr (after a galaxy crosses the shock front), once the $\mathrm{O}$ stars no longer significantly contribute to the stellar population. A timescale of $10 \mathrm{Myr}$ corresponds roughly to $75 \mathrm{kpc}$ in Figure 3. On such short timescales, it is difficult to detect an excess of any particular galaxy type because of small number statistics (i.e.- there are not enough galaxies within a single $75 \mathrm{kpc}$ bin). Also, post-starburst galaxies are difficult to distinguish from quiescent elliptical galaxies with photometry, since both populations lie near the optical CMR (Tran et al. 2007).

However, the lifetime of a post-starburst galaxy is 1 Gyr (e.g. Quintero et al. 2004), providing us with a longer timescale (or distance from the shock front) in which we can search for an excess of these galaxy types. In future work, we will spectroscopically identify the $\mathrm{E}+\mathrm{A}$ galaxies in our sample. A higher fraction of $\mathrm{E}+\mathrm{A}$ galaxies behind the shock front versus ahead of the shock front, coupled with no change in current star formation rates across the shock boundary, would favor ram pressure stripping of gas reservoirs, rather than some effect on current star formation.

Although Figure 3 shows that there is no significant change in color in the pre-shock versus post-shock galaxies, the geometric effects and contamination from noncluster sources may dilute the signal. Future work includes analysis of IRAC and MIPS $24 \mu \mathrm{m}$ data observed over a wider area, which coupled with spectroscopic redshifts will be less sensitive to geometric effects and contamination. However, even with our current level of contamination, it is clear that the ram pressure exerted from the supersonic gas does not have a dramatic impact on current star formation activity in the main cluster galaxies.

Another source of signal dilution is the large fraction of early-type galaxies in our sample, for which there are two possible explanations. First, galaxy preprocessing in the group environment may have already done its work on both the cluster and subcluster galaxies, explaining the observed lack of late-type galaxies (Zabludoff \& Mulchaey 1998; Cortese et al. 2006). Second, the galaxies we observe may have been affected by the cluster environment in such a manner that they have already converted their gas reservoir into stars. Marcillac et al. (2007) and Bai et al. (2007) have investigated star formation of galaxies in merging clusters at $z \sim 0.83$. They find evidence of triggered star formation in infalling galaxies and galaxy groups, which coupled with mechanisms such as ram pressure stripping or galaxy harassment, can act to eventually quench star formation. In this scenario, many of the initially gas-rich, star forming galaxies in the main cluster would have consumed much of their available gas in an earlier epoch of triggered star formation, explaining the lack of late-type galaxies in our current sample. However, the relative lack of late-type galaxies cannot explain why we do not see a signal across the shock front, especially considering that even among the late-type population, we do not detect 
a significant difference in color in the post-shock versus pre-shock galaxies.

\section{SUMMARY}

To trace specific star formation rate, we use IRAC color $\mathrm{m}_{4.5}-\mathrm{m}_{8}$. The $8 \mu \mathrm{m}$ flux traces star formation via the $\mathrm{PAH}$ emission feature at rest wavelength $6.2 \mu \mathrm{m}$, which is redshifted into the $8 \mu \mathrm{m}$ band at the cluster redshift. The $4.5 \mu \mathrm{m}$ flux can be taken as a proxy for stellar mass.

Using spectroscopic redshifts, we confirmed 63 out of 200 IRAC sources (169 in direct path of shock front + 31 above/below the shock front) as cluster members, and found 42 IRAC sources that are interlopers. Applying a magnitude cut at $\mathrm{m}_{4.5}=17$, we are left with 158 cluster candidates, of which 134 are in the direct path of the shock front.

We observe no significant trend in $\mathrm{m}_{4.5}-\mathrm{m}_{8}$ as a function of distance from the shock front. The KS-test reveals that the sample of galaxies behind the shock versus ahead of the shock front only differs in color by less than $1.5 \sigma$, for galaxies with various cuts in $\mathrm{m}_{4.5}-\mathrm{m}_{8}$ color. This indicates that even in a dramatic merger event like the Bullet Cluster, the ram pressure induced from a supersonic collision does not drastically trigger or quench current/recent star formation. A possible explanation is that the ram pressure can remove the gas reservoirs in the outer disk, while preserving star formation in the central disk of a galaxy. While geometric effects, contamination from interlopers, and the lack of late-type galaxies in the Bullet Cluster could contribute to diluting a signal, they are unlikely to explain the complete lack of signal we observe. Even among our small sample of late-type galaxies, we detect no change in color between the pre-shock and post-shock galaxies to within $1.5 \sigma$.

The authors would like to thank Rafael Barrena for sharing his redshift data with us. We also acknowledge support for this work from NASA/Spitzer grant 1319141. MM was supported by NASA contract NAS839073 and Chandra grant GO8-9128X. Christine Jones acknowledges support from Spitzer Contract 1265584.

Facilities: Spitzer (IRAC), CXO (ACIS-I), Magellan:Baade (IMACS) 


\section{REFERENCES}

Allamandola, L. J., Tielens, A. G. G. M., \& Barker, J. R. 1985, ApJ, 290, L25

Assef, R. J., et al. 2008, ApJ, 676, 286

Bai, L., et al. 2007, ApJ, 664, 181

Barrena, R., Biviano, A., Ramella, M., Falco, E. E., \& Seitz, S. 2002, A\&A, 386, 816

Bekki, K., Couch, W. J., \& Shioya, Y. 2002, ApJ, 577, 651

Berrier, J. C., Stewart, K. R., Bullock, J. S., Purcell, C. W. Barton, E. J., \& Wechsler, R. H. 2008, ArXiv e-prints, 804, arXiv:0804.0426

Boselli, A., Lequeux, J., \& Gavazzi, G. 2004, A\&A, 428, 409

Boselli, A., \& Gavazzi, G. 2006, PASP, 118, 517

Bertin, E., \& Arnouts, S. 1996, A\&AS, 117, 393

Bigelow, B. C., Dressler, A. M., Shectman, S. A., \& Epps, H. W. 1998, Proc. SPIE, 3355, 225

Brandl, B. R., et al. 2006, ApJ, 653, 1129

Brüggen, M., \& De Lucia, G. 2008, MNRAS, 383, 1336

Chung, A., van Gorkom, J. H., Kenney, J. D. P., \& Vollmer, B. 2007, ApJ, 659, L115

Clowe, D., Bradač, M., Gonzalez, A. H., Markevitch, M., Randall, S. W., Jones, C., \& Zaritsky, D. 2006, ApJ, 648, L109

Cortese, L., Gavazzi, G., Boselli, A., Franzetti, P., Kennicutt, R. C., O’Neil, K., \& Sakai, S. 2006, A\&A, 453, 847

Cortese, L., et al. 2007, MNRAS, 376, 157

Dressler, A. 1980, ApJ, 236, 351

Engelbracht, C. W., Gordon, K. D., Rieke, G. H., Werner, M. W. Dale, D. A., \& Latter, W. B. 2005, ApJ, 628, L29

Freudling, W., Siebenmorgen, R., \& Haas, M. 2003, ApJ, 599, L13

Gavazzi, G., Boselli, A., Mayer, L., Iglesias-Paramo, J., Vílchez, J. M., \& Carrasco, L. 2001, ApJ, 563, L23

Gavazzi, G., Cortese, L., Boselli, A., Iglesias-Paramo, J., Vílchez, J. M., \& Carrasco, L. 2003, ApJ, 597, 210

Gunn, J. E., \& Gott, J. R. I. 1972, ApJ, 176, 1

Howell, J. H., et al. 2007, AJ, 134, 2086

Fazio, G. G., et al. 2004, ApJS, 154, 10

Kawata, D., \& Mulchaey, J. S. 2008, ApJ, 672, L103

Kennicutt, R. C., Jr. 1998, ARA\&A, 36, 189

Kodama, T., Smail, I., Nakata, F., Okamura, S., \& Bower, R. G. 2001, ApJ, 562, L9

Kronberger, T., Kapferer, W., Ferrari, C., Unterguggenberger, S., \& Schindler, S. 2008, A\&A, 481, 337
Lacy, M., et al. 2004, ApJS, 154, 166

Leger, A., \& Puget, J. L. 1984, A\&A, 137, L5

Li, A., \& Draine, B. T. 2002, ApJ, 572, 232

López-Cruz, O., Barkhouse, W. A., \& Yee, H. K. C. 2004, ApJ, 614,679

Marcillac, D., Rigby, J. R., Rieke, G. H., \& Kelly, D. M. 2007, ApJ, 654,825

Markevitch, M., Gonzalez, A. H., David, L., Vikhlinin, A., Murray, S., Forman, W., Jones, C., \& Tucker, W. 2002, ApJ, 567, L27

Markevitch, M., Gonzalez, A. H., Clowe, D., Vikhlinin, A., Forman, W., Jones, C., Murray, S., \& Tucker, W. 2004, ApJ, 606, 819

Mayer, L., Mastropietro, C., Wadsley, J., Stadel, J., \& Moore, B. 2006, MNRAS, 369, 1021

Milosavljević, M., Koda, J., Nagai, D., Nakar, E., \& Shapiro, P. R 2007, ApJ, 661, L131

Moore, B., Katz, N., Lake, G., Dressler, A., \& Oemler, A. 1996, Nature, 379, 613

Navarro, J. F., Frenk, C. S., \& White, S. D. M. 1997, ApJ, 490, 493

Peeters, E., Spoon, H. W. W., \& Tielens, A. G. G. M. 2004, ApJ, 613,986

Pizagno, J., et al. 2005, ApJ, 633, 844

Quintero, A. D., et al. 2004, ApJ, 602, 190

Roediger, E., \& Hensler, G. 2005, A\&A, 433, 875

Smith, J. D. T., et al. 2007, ApJ, 656, 770

Springel, V., \& Farrar, G. R. 2007, MNRAS, 380, 911

Stern, D., et al. 2005, ApJ, 631, 163

Tran, K.-V. H., van Dokkum, P., Illingworth, G. D., Kelson, D. Gonzalez, A., \& Franx, M. 2005, ApJ, 619, 134

Tran, K.-V. H., Franx, M., Illingworth, G. D., van Dokkum, P., Kelson, D. D., Blakeslee, J. P., \& Postman, M. 2007, ApJ, 661, 750

Uchida, K. I., Sellgren, K., \& Werner, M. 1998, ApJ, 493, L109

Vollmer, B., Cayatte, V., Balkowski, C., \& Duschl, W. J. 2001, ApJ, 561, 708

Vollmer, B., Soida, M., Chung, A., van Gorkom, J. H., Otmianowska-Mazur, K., Beck, R., Urbanik, M., \& Kenney, J. D. P. 2008, A\&A, 483, 89

Zabludoff, A. I., \& Mulchaey, J. S. 1998, ApJ, 496, 39

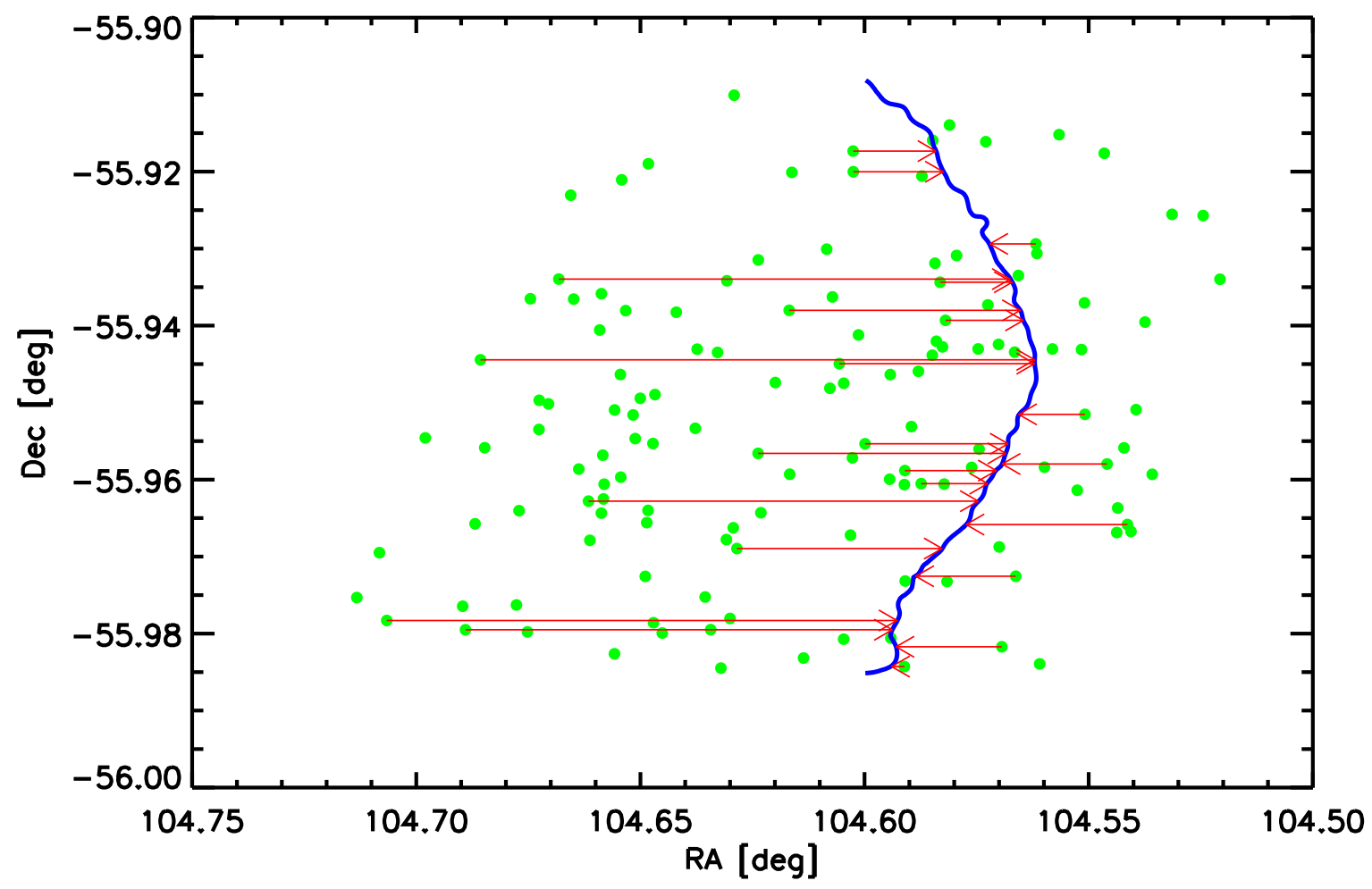

Fig. 1. - Bullet Cluster candidates (filled circles) shown with the shock front (a corresponding contour of constant X-ray surface brightness). Arrows indicate the distance between the object and the shock front for sources with $\mathrm{IRAC}_{\mathrm{A}}$ color $\mathrm{m}_{4.5}-\mathrm{m}_{8}>0.5$. 




FIG. 2.- The $\mathrm{m}_{4.5}-\mathrm{m}_{8}$ color as a function of distance from the shock front for 134 cluster candidates. The lower and upper axes indicate projected distance in degrees and Mpc, respectively. Spectroscopically confirmed cluster members are filled in boxes. The vertical dotted line shows the location of the shock front. Sources to the left of this line are considered post-shock galaxies, and those to the right are considered pre-shock galaxies. The horizontal dotted-dashed lines at $\mathrm{m}_{4.5}-\mathrm{m}_{8}=-0.13$ and $\mathrm{m}_{4.5}-\mathrm{m}_{8}=2.43$ represent the expected color of an Elliptical and Sbc galaxy at $\mathrm{z}=0.3$, respectively (Assef et al. 2008). The error bar in the upper left corner shows the mean color error of the 134 candidates. 


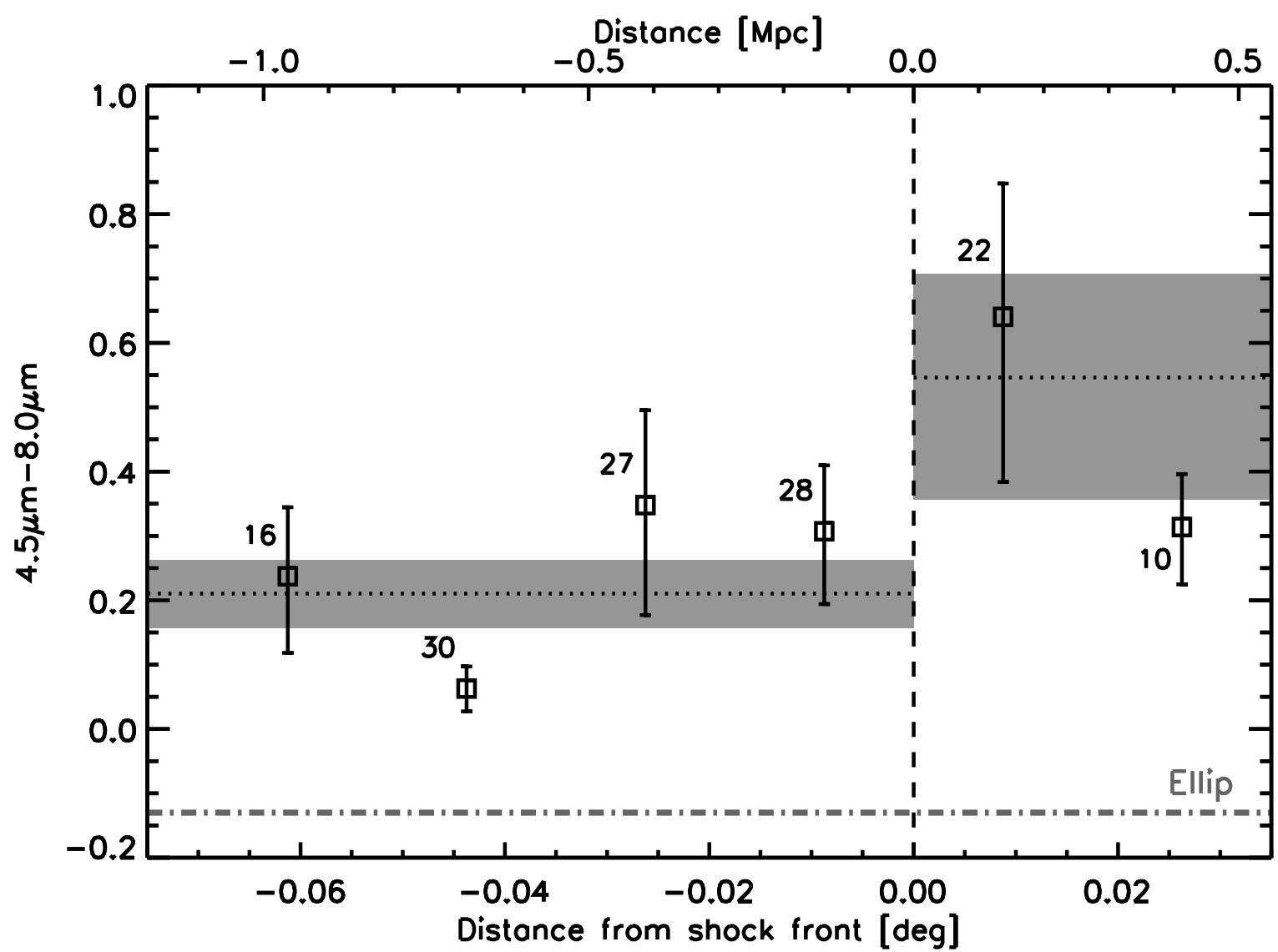

FIG. 3.- The $\mathrm{m}_{4.5}-\mathrm{m}_{8}$ color as a function of distance from shock front for cluster candidates in bins of $275 \mathrm{kpc}$, with the number of sources per bin denoted. Individual error bars are derived from bootstrapping method. Grey shaded regions show the integrated color for all objects behind and ahead of the shock front, $\pm 1 \sigma$ derived from bootstrapping. 


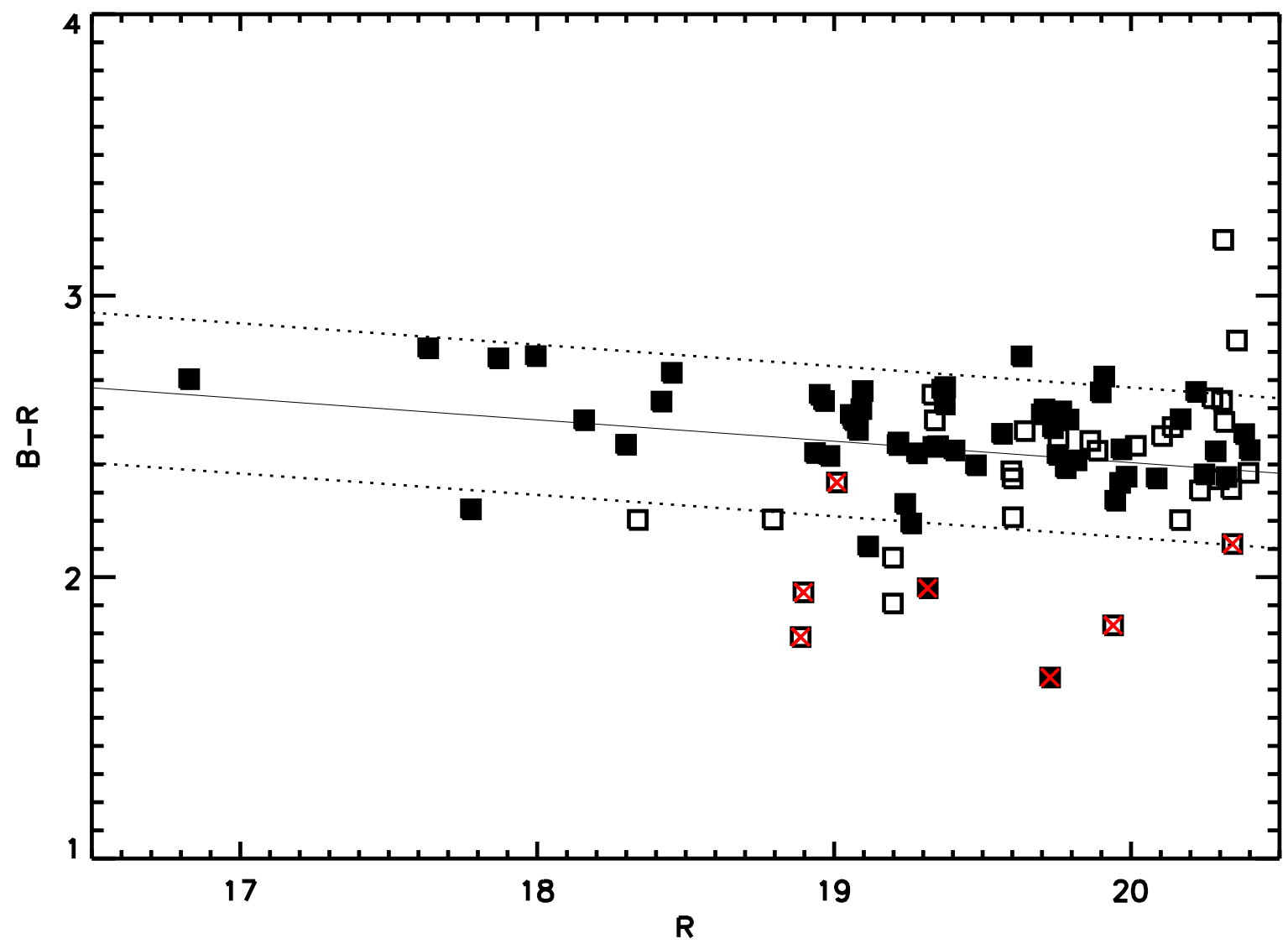

FIG. 4. - The optical color-magnitude diagram shown for 88 IRAC selected cluster candidates. The solid line is the color-magnitude relation whose slope and zero-point were adopted from López-Cruz et al. (2004). The dotted lines signify $2 \sigma$ from the fit, where the dispersion is obtained by fitting a Gaussian to the residuals. Objects overplotted with a cross symbol have IRAC color $\mathrm{m}_{4.5}-\mathrm{m}_{8}>0.5$ and are thus likely to be star-forming galaxies. 